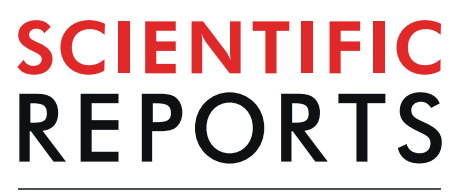

natureresearch

\title{
Lifetime risks, expected years of life lost, and cost-per-life year of esophageal cancer in Taiwan
}

\begin{abstract}
Wu-Wei Lai ${ }^{1}$, Chia-Ni Lin ${ }^{2}$, Chao-Chun Chang ${ }^{1}$ \& Jung-Der Wang ${ }^{2,3 *}$
Besides lifetime risks, we estimated life expectancy (LE), expected years of life lost (EYLL), lifetime costs, and cost-per-LY (life-year) stratified by different stages of esophageal cancer (EC). From the Taiwan Cancer Registry, we collected 14,420 EC to estimate the incidence rates during 2008-2014. They were followed to 2015 to obtain the survival function, which was extrapolated to lifetime by a semiparametric method. We abstracted the monthly healthcare expenditures from the reimbursement database of National Health Insurance, which were multiplied with the corresponding survival probabilities to quantify lifetime cost and cost-per-LY after adjustments. About $93.7 \%$ of patients were male; $90.8 \%$ had squamous cell carcinoma. Most patients were diagnosed at advanced stages, with $44.6 \%$ and $28.3 \%$ at stages III and IV. The lifetime risk of EC in males increased in Taiwan with a cumulative incidence rate $\left(\mathrm{CIR}_{30-84}\right)$ of $0.0146 \%$ (2008 2010) to $0.0165 \%$ (2013-2014). The EYLL for stages I-IV were $15.8,17.5,20.5$, and 22.5, while the average of cost-per-LY for stages I-IV were US\$ $6,987, \$ 8,776, \$ 12,153$, and $\$ 22,426$. EC in Taiwan appears to have shifted into younger ages groups and incidence is still increasing. Strategies for prevention, early diagnosis and treatment are warranted to improve the cost-effectiveness and control of this cancer.
\end{abstract}

In Taiwan, esophageal cancer was the $9^{\text {th }}$ leading cause of cancer death in $2016^{1-3}$, which is similar to other areas of world ${ }^{4}$. Most esophageal cancer patients are male and diagnosed at advanced stages, which usually result in short survival and heavy financial burdens on the individual, family, and society ${ }^{5}$. For cancer control, stakeholders are generally interested in the following indicators: incidence rates (IR), life expectancies (LEs) after diagnosis, expected years of life lost (EYLL, i.e. loss of life expectancy), and lifetime health care expenditures ${ }^{6,7}$. If advanced esophageal cancer patients could be detected at an earlier stage by emerging innovations, such as novel circular RNA (CircRNAs) ${ }^{8,9}$, the potential benefits of reducing EYLL, lifetime cost, and productivity loss, etc. would create additional incentives for cancer screening and treatment ${ }^{6,10}$.

In Taiwan, the National Health Insurance (NHI) was founded in 1995, and by 2009 more than $99 \%$ of people in Taiwan were covered with full reimbursement ${ }^{11,12}$. All of the reimbursement-related data of the NHI have been edited and maintained by the National Health Research Institutes (NHRI) and Department of Statistics of the Ministry of Health and Welfare. Since there are relatively few studies addressing healthcare costs and outcomes from a lifetime horizon for esophageal cancer, it is worthwhile to tackle the above challenges from our nationwide large scale data stratified by stage. Through retrieving and analyzing the above national dataset, the aim of this study was to determine the lifetime risks, LEs after diagnosis, EYLL, lifetime cost, and cost-effectiveness ratio (CER) of esophageal cancer in Taiwan from 2008 to 2014 with a total number of 14,420 patients stratified by 4 stages.

\section{Results}

The mean age at diagnosis was younger for males than females (58.0v.s. 65.2). Esophageal cancer in Taiwan is still more predominant in male (93.7\%), and $90.8 \%$ are squamous cell carcinoma (Table 1). The incidence of squamous esophageal cancer in males increased gradually in Taiwan with a $\mathrm{CIR}_{30-84}(\%)$ of 0.146 (2008 2010) to 0.165 (2013-2014) (Tables 1 and 2). A shift in the highest IR to a younger age-group from 60-69y/o in 2008-2010 to 50-59y/o in 2013-2014 was also noted (Table 2 and supplementary figure). Although diagnosis at earlier stages appears to have increased slightly

${ }^{1}$ Department of Surgery, National Cheng Kung University Hospital, College of Medicine, National Cheng Kung University, Tainan, Taiwan. ${ }^{2}$ Department of Public Health, National Cheng Kung University College of Medicine, Tainan, Taiwan. ${ }^{3}$ Department of Internal Medicine and Occupational and Environmental Medicine, National Cheng Kung University Hospital, College of Medicine, National Cheng Kung University, Tainan, Taiwan. *email: jdwang121@gmail.com 


\begin{tabular}{|c|c|}
\hline & The national cohort \\
\hline Total number of patients & 14,420 \\
\hline 2008 & 1,657 \\
\hline 2009 & 1,848 \\
\hline 2010 & 2,055 \\
\hline 2011 & 2,065 \\
\hline 2012 & 2,171 \\
\hline 2013 & 2,263 \\
\hline 2014 & 2,361 \\
\hline \multicolumn{2}{|l|}{ Sex, no. of patients (\%) } \\
\hline Male/Female & $13,515(93.7) / 905(6.3)$ \\
\hline \multicolumn{2}{|c|}{ Age at diagnosis, mean $(\mathrm{SD})$ years } \\
\hline Male/Female & $58.0(11.7) / 65.2(14.1)$ \\
\hline \multicolumn{2}{|c|}{ Age distribution, no of patients (\%) } \\
\hline$<50$ & $3,471(24.1)$ \\
\hline $50 \sim 64$ & $6,992(48.5)$ \\
\hline$\geq 65$ & $3,957(27.4)$ \\
\hline \multicolumn{2}{|c|}{ Histology, no. of patients (\%) } \\
\hline Squamous & $13,088(90.8)$ \\
\hline Adenocarcinoma & $476(3.3)$ \\
\hline Others & $856(5.9)$ \\
\hline \multicolumn{2}{|c|}{ Sequence of cancer diagnosed, no of patients (\%) } \\
\hline 1 & $11,803(81.9)$ \\
\hline$>1$ & $2,617(18.1)$ \\
\hline \multicolumn{2}{|l|}{ Stage, no. of patients (\%) } \\
\hline No information & $1,923(13.3)$ \\
\hline With staging information & $12,497(86.7)$ \\
\hline 0 & $211(1.7)$ \\
\hline I & $1,111(8.9)$ \\
\hline II & $2,064(16.5)$ \\
\hline III & 5,575 (44.6) \\
\hline IV & $3,536(28.3)$ \\
\hline
\end{tabular}

Table 1. Demographic characteristics of esophageal cancer patients in Taiwan, 2008-2014.

\begin{tabular}{|c|c|c|c|c|c|c|c|c|c|c|c|c|c|}
\hline \multirow[b]{2}{*}{ Histology } & \multirow{2}{*}{$\begin{array}{l}\text { Year- } \\
\text { period }\end{array}$} & \multicolumn{12}{|l|}{ Age } \\
\hline & & $30-34$ & $35-39$ & $40-44$ & $45-49$ & 50-54 & $55-59$ & $60-64$ & $65-69$ & $70-74$ & 75-79 & $80-84$ & $\mathrm{CIR}_{30-84}(\%)$ \\
\hline \multirow{3}{*}{$\begin{array}{l}\text { Sqamous cell } \\
\text { carcinoma }\end{array}$} & $2008-10$ & 0.6 & 5.1 & 15.1 & 24.6 & 33.9 & 38.0 & $\underline{39.9}$ & $\underline{40.3}$ & 36.7 & 31.0 & 29.5 & 0.146 \\
\hline & 2011-12 & 0.6 & 4.6 & 14.7 & 28.3 & 37.5 & $\underline{43.2}$ & $\underline{42.7}$ & 42.6 & 37.0 & 34.3 & 28.1 & 0.156 \\
\hline & 2013-14 & 0.7 & 3.9 & 15.6 & 30.9 & $\underline{43.8}$ & $\underline{45.0}$ & 42.3 & 39.7 & 39.1 & 38.6 & 33.0 & 0.165 \\
\hline \multirow{3}{*}{ Adeno-carcinoma } & $2008-10$ & 0.1 & 0.6 & 0.9 & 1.8 & 2.3 & 3.6 & 4.0 & 4.4 & 6.2 & 4.9 & 7.7 & 0.018 \\
\hline & $2011-12$ & 0.2 & 0.3 & 1.0 & 1.8 & 3.0 & 4.3 & 3.7 & 3.9 & 3.9 & 5.4 & 6.9 & 0.017 \\
\hline & 2013-14 & 0.2 & 0.4 & 0.8 & 1.7 & 3.1 & 3.4 & 4.0 & 3.9 & 5.0 & 4.1 & 9.6 & 0.018 \\
\hline
\end{tabular}

Table 2. Incidence rate (IR, $1 / 10^{5}$ person-years) and cumulative incidence rate $\left[\mathrm{CIR}_{30-84}(\%)\right]$ of male esophageal cancer in Taiwan stratified by histology and 5-year ages group.

in recent years (Table 3), most patients were diagnosed at advanced stages, with $44.6 \%$ and $28.3 \%$ at stages III and IV, respectively (Tables 1 and 3). However, the above findings were not the case for Taiwan esophageal adenocarcinoma. Table 4 summarizes the LE after diagnosis, EYLL, lifetime costs, and cost per life year of esophageal cancer. In general, the more advanced the stage, the shorter the LE and the higher the EYLL (Fig. 1). In contrast, the cost per life year of esophageal cancer increased along with advanced stage, while lifetime costs showed a decreasing trend. Patients with adenocarcinoma were diagnosed at an older age but survival was similar to those of squamous cell carcinoma, which resulted in a smaller loss-of-LE for the former and similar lifetime costs and cost-effectiveness ratios for both.

\section{Discussion}

Lifetime risks and incidence rates: What's new? Our study shows that esophageal cancer in Taiwan is still a male (93.7\%), and squamous cell carcinoma (90.8\%) predominant disease, mostly diagnosed at advanced stages, with $44.6 \%$ and $28.3 \%$ at stages III and IV, respectively. The above findings corroborate with previous reports $^{2,3,13-17}$. We calculated the cumulative incidence rate, which is the probability of an event occurring during 


\begin{tabular}{|c|c|c|c|c|c|c|c|c|c|}
\hline \multirow[b]{3}{*}{ Stage } & \multirow[b]{3}{*}{ Yr period } & \multicolumn{8}{|c|}{ Squamous } \\
\hline & & \multicolumn{4}{|c|}{ Male case No. } & \multicolumn{3}{|c|}{ IR ( $\left(1 / 10^{5}\right.$ person-years $)$} & \multirow{2}{*}{\begin{tabular}{|l|} 
Lifetime risk \\
$\operatorname{CIR}_{30-84}\left(1 / 10^{5}\right)$ \\
\end{tabular}} \\
\hline & & Total & $30-49$ & $50-64$ & 65-84 & $30-49$ & $50-64$ & $65-84$ & \\
\hline \multirow{3}{*}{$0^{*}$} & $2008-10$ & 41 & 13 & 16 & 12 & 0.11 & 0.27 & 0.37 & \begin{tabular}{|l|}
0.137 \\
\end{tabular} \\
\hline & 2011-12 & 53 & 21 & 25 & 7 & 0.28 & 0.55 & 0.32 & 0.204 \\
\hline & 2013-14 & 68 & 17 & 38 & 13 & 0.22 & 0.79 & 0.58 & 0.279 \\
\hline \multirow{3}{*}{ I } & 2008-10 & 322 & 97 & 145 & 80 & 0.86 & 2.40 & 2.49 & 1.029 \\
\hline & 2011-12 & 281 & 96 & 135 & 50 & 1.27 & 3.00 & 2.32 & 1.166 \\
\hline & 2013-14 & 353 & 95 & 201 & 57 & 1.26 & 4.17 & 2.55 & 1.386 \\
\hline \multirow{3}{*}{ II } & $2008-10$ & 747 & 180 & 361 & 206 & 1.59 & 5.98 & 6.41 & 2.494 \\
\hline & 2011-12 & 485 & 121 & 249 & 115 & 1.60 & 5.53 & 5.34 & 2.213 \\
\hline & 2013-14 & 514 & 133 & 251 & 130 & 1.76 & 5.21 & 5.81 & 2.293 \\
\hline \multirow{3}{*}{ III } & $2008-10$ & 1623 & 493 & 772 & 358 & 4.35 & 12.80 & 11.14 & 5.006 \\
\hline & $2011-12$ & 1586 & 378 & 842 & 366 & 4.99 & 18.69 & 16.99 & 7.173 \\
\hline & 2013-14 & 1704 & 418 & 931 & 355 & 5.53 & 19.32 & 15.87 & 7.153 \\
\hline \multirow{3}{*}{ IV } & $2008-10$ & 1441 & 385 & 714 & 342 & 3.40 & 11.84 & 10.64 & 4.573 \\
\hline & 2011-12 & 718 & 180 & 391 & 147 & 2.37 & 8.68 & 6.82 & 3.137 \\
\hline & 2013-14 & 775 & 166 & 440 & 169 & 2.20 & 9.13 & 7.56 & 3.315 \\
\hline
\end{tabular}

Table 3. Number of new cases, incident Rate $\left(1 / 10^{5}\right.$ person-years) and lifetime cumulative incidence rate $\left[\operatorname{CIR}_{30-84}\left(1 / 10^{5}\right)\right]$ of esophageal squamous cell carcinoma in males stratified by stage and period of calendar years. * Stage $0: \mathrm{T}_{\mathrm{is}} \mathrm{N}_{0} \mathrm{M}_{0}$; $\mathrm{T}_{\mathrm{is}}$ : carcinoma in situ.

\begin{tabular}{|c|c|c|c|c|c|c|}
\hline Category & stage & $\begin{array}{l}\text { Onset age } \\
\text { mean } \pm S D \text { years }\end{array}$ & $\begin{array}{l}\text { LE, mean } \\
\text { (SE) years }\end{array}$ & $\begin{array}{l}\text { EYLL mean } \\
\text { (SE) years }\end{array}$ & $\begin{array}{l}\text { lifetime costs (SE) } \\
\text { [3\% discount, USD] }^{a}\end{array}$ & $\begin{array}{l}\text { CER, Cost/LE } \\
\text { (SE) [USD] }\end{array}$ \\
\hline \multirow{5}{*}{ Male } & Stage I $(\mathrm{n}=1,038)$ & $56.9 \pm 11.3$ & $8.5(1.8)$ & $15.8(1.8)$ & $52,096(4,153)$ & $6,987(551)$ \\
\hline & Stage II $(\mathrm{n}=1,895)$ & $58.7 \pm 12.3$ & $5.4(1.0)$ & $17.5(1.0)$ & $42,579(1,937)$ & $8,776(769)$ \\
\hline & Stage III $(\mathrm{n}=5,257)$ & $57.5 \pm 11.4$ & $3.2(0.3)$ & $20.5(0.3)$ & $35,381(820)$ & $12,153(648)$ \\
\hline & Stage IV $(\mathrm{n}=3,344)$ & $57.9 \pm 11.4$ & $1.0(0.1)$ & $22.5(0.2)$ & $20,907(388)$ & $22,426(998)$ \\
\hline & Unknown $(\mathrm{n}=1,786)$ & $59.6 \pm 12.6$ & $2.9(0.4)$ & $19.4(0.4)$ & $30,778(1,095)$ & $11,392(837)$ \\
\hline \multirow{2}{*}{ Female } & Stage I+II $(\mathrm{n}=242)$ & $65.2 \pm 13.7$ & $5.5(1.7)$ & $16.0(1.7)$ & $37,960(5,128)$ & $7,468(1,121)$ \\
\hline & Stage III+IV $(\mathrm{n}=510)$ & $64.3 \pm 14.3$ & $1.9(0.4)$ & $20.4(0.6)$ & $25,331(1,418)$ & $13,686(1,548)$ \\
\hline \multicolumn{2}{|c|}{ Adenocarcinoma $(n=476)$} & $66.4 \pm 14.4$ & $2.6(0.6) *$ & $15.5(0.7)^{* *}$ & $27,047(2,276)$ & $11,015(1,172)$ \\
\hline \multicolumn{2}{|c|}{ Squamous cell carcinoma $(\mathrm{n}=13,088)$} & $58.0 \pm 11.7$ & $3.4(0.3)^{*}$ & $20.2(0.3)^{* *}$ & $34,711(960)$ & $10,604(497)$ \\
\hline
\end{tabular}

Table 4. The LE (life expectancy), EYLL (expected years of life lost), Lifetime healthcare expenditures (in US dollars) and cost-per-life year of esophageal cancer stratified by sex, stage, and histology. ${ }^{\text {a Lifetime costs }}$ in US dollars a exchange rate on 2015/12/31 ( 1 USD $=33.017$ NTD) provided by Bank of Taiwan. ${ }^{*} \mathrm{p}=0.21$; $* * \mathrm{p}=0.000$

a stated period of observation, if the person has not died of other diseases. Therefore, the $\left[\mathrm{CIR}_{30-84}(\% 0)\right]$ can be interpreted as the lifetime risk of disease occurrence ${ }^{18}$. We found that the incidence of squamous esophageal cancer in males in Taiwan continuously increased with a $\mathrm{CIR}_{30-84}$ of $0.0146 \%$ (2008 2010) to $0.0165 \%$ (2013-2014) (Table 2). Although the same increasing trend had been reported previously ${ }^{3,4,17}$, they were age-standardized incidence rates and may not be easily understood by lay people. In contrast, our figures can be interpreted as the lifetime probability of developing esophageal cancer for Taiwanese males.

In addition, our findings (Table 2 and Supplementary Fig. 1) revealed a slight but consistent shift in the highest incidence rate toward a younger age-group, namely, from 60-69y/o in 2008-2010 to 50-59y/o in 2013-2014 in males with squamous esophageal cancer in Taiwan. Accordingly, the mean age at diagnosis of squamous esophageal cancer in Taiwanese males moved from $60(1998 \sim 2007)^{13}$, and 59.1(1998-2012) years old ${ }^{19}$, down to 58 y/o (2008 2014) (Table 1). We conjectured that such a slight shift could be partially explained by an increased proportion of younger patients diagnosed at stage 3 in comparison to stage 4 in recent years (calendar periods of 2008-10 v.s.2011-2 and 2013-4, in Table 3). More studies are needed to explore detailed reasons for this phenomenon in the future.

An indicator of prognosis: life expectancy. Survival rates are a measure of how many people remain alive with cancer after a certain period of time. For example, the 5 - yr survival rates were $44.2 \%$ for clinical stage I (cI) of esophageal cancer patients in Taiwan; $27.5 \%$ for cII; $15.6 \%$ for cIII; and 3.4\% for cIV as reported by Cheng YF et al. ${ }^{17}$. However, not everyone living with cancer is interested in hearing about statistics on survival rates. More people hope to know how long they could live with a particular type of cancer. Therefore, life expectancy (LE) would be a viable alternative when informing patients, i.e., the number of years that someone is expected 
Expected years of life lost (EYLL) of male and female esophageal cancer

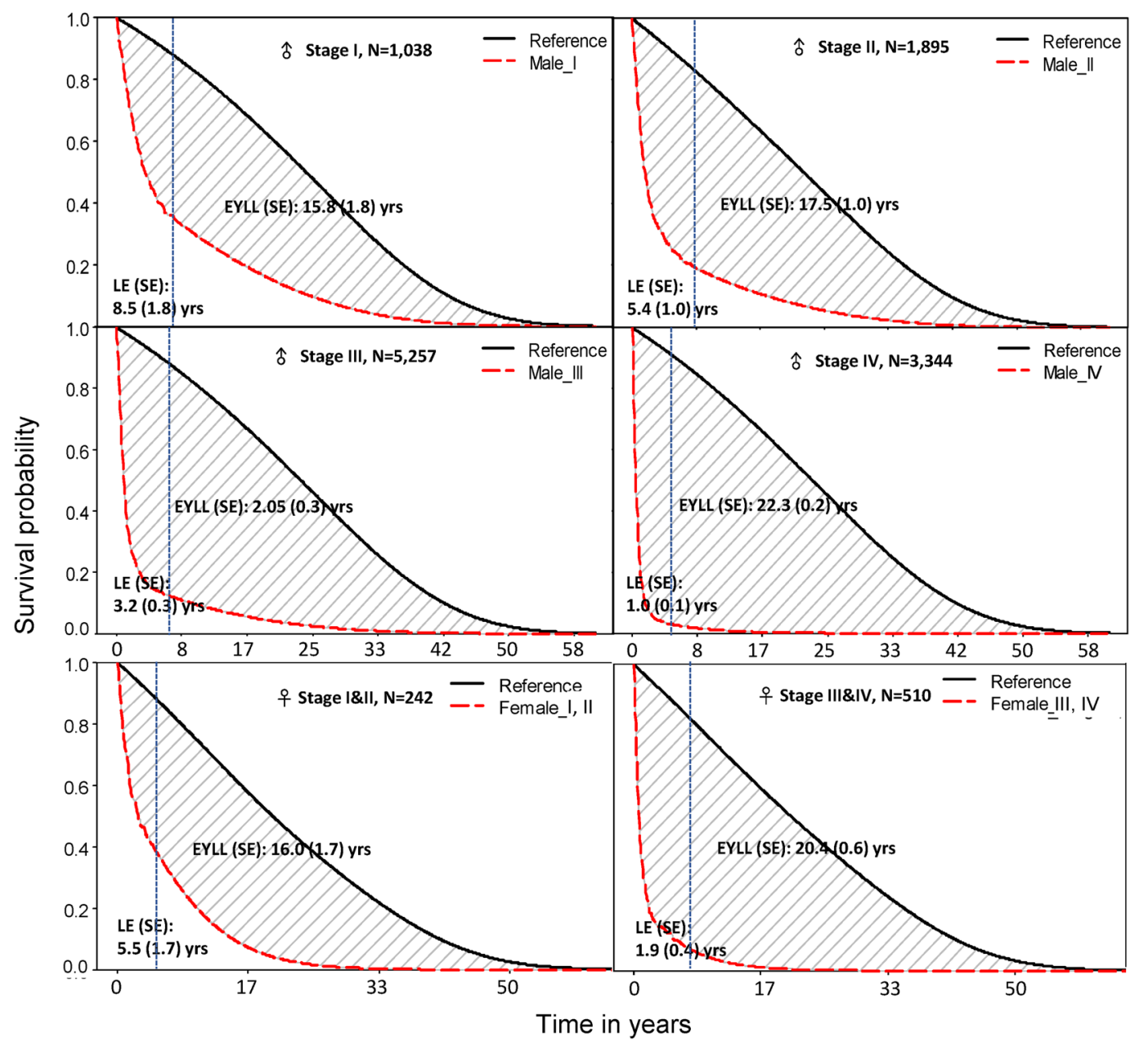

Figure 1. Life expectancies (LE) of esophageal cancer patients and their age- and sex-matched referents stratified by sex and stage: Shaded regions indicate the expected years of life loss (EYLL), or, loss of life expectancy that could be saved by prevention. The dotted blue line on each panel indicates the end of follow-up which is the beginning of rolling (month-by-month) extrapolation of the cancer cohort.

to live from the date of diagnosis. In general, LE is more relevant to patients for the future planning of their daily lives. In this study, the LEs of each stage for squamous esophageal cancer in male patients in Taiwan were as follows: 8.5 yrs for stage I; 5.4 for II; 3.2 for III; 1.0 for IV; and 2.9 for unknown stage (Table 4 and Fig. 1). The LE of esophageal cancer without staging information in Taiwan (1998 2012) was 3.1 years, as reported by Wu TY et $a l .{ }^{19}$, indicating very limited or no improvement in prognosis. Regarding the prognosis of different pathological types, we found that the loss-of-LE of patients with adenocarcinoma was significantly lower than that of squamous cell carcinoma, which corroborate with previous reports ${ }^{20,21}$. Future studies are warranted to explore the distinctions between these two pathological types.

Indicators of societal burdens: Expected years of life loss and lifetime cost. Expected years of life lost (EYLL) is an estimate of the average years a person would have lived if he or she had not died prematurely ${ }^{22}$. It is therefore, a more accurate measure of premature mortality similar to deaths before 65 years old ${ }^{23}$. Our results showed that esophageal cancers is a disease with high premature death with $>15$ years of EYLL, even at the earliest stages for both males and females, 15.8 and 16.0 years of EYLL, respectively (Table 4 and Fig. 1). This implies that the unhealthy lifestyle (cigarette smoking, drinking alcohol, and betel quid chewing) of esophageal cancer patients can have a big impact on their remaining life. As stated by Ferronha l et al. and Kuang JJ et al., many cancer survivors continue smoking and /or drinking even after diagnosis, which may result in a high cumulative exposures and poor survival ${ }^{24-26}$. As many as $27 \%$ of esophageal cancer patients have a chances of developing metachronous second primary cancer stated by Chen MF et al. ${ }^{13}$ in a study of a Taiwan nationwide esophageal cancer database from 1998-2007. The percentage of synchronous cancers diagnosed at the same time as esophageal cancer was around 4.1-4.5\% ${ }^{27,28}$. The percentage of antecedent tumors of esophageal cancer, i.e. esophageal cancer as a second primary, following first primary cancers of other sites in this study was $18.1 \%$ (Table 1). The EYLL of male esophageal patients with unknown staging was 19.4 in this study. Our finding was in line with that 
(19.1) reported by Wu TY et al. ${ }^{19}(1998-2012)$. Therefore, primary prevention for avoiding known risk factors, such as cigarette smoking, drinking alcohol, and betel quid chewing, might be the best strategy to reduce morbidity and mortality from esophageal cancer ${ }^{29,30}$.

Another relevant indicator of societal burden is the lifetime spending for healthcare services of cancer, as summarized in Table 4. Lifetime cost for unknown staging of esophageal cancer in Taiwan male patients was US\$ 30,778 , which seems to be consistent with that (US\$ 25,900) reported by Wu TY et al. ${ }^{19}$. In the typical studies of cost-effectiveness, researcher hope to quantify the incremental cost-effectiveness ratio (ICER), which is an overall measure representing the economic value of an intervention, compared with an alternative (comparator) ${ }^{31}$. An ICER is calculated by dividing the difference in total costs (incremental cost) by the difference in the chosen measure of health outcome or effect (incremental effect) to provide a ratio of "extra cost per extra unit of health effect" - for the more expensive therapy vs the alternative. Here, we considered both lifetime costs and LY (life-year) together as a cost effectiveness ratio (CER, or. Lifetime cost/LY, with a discounting rate adjusted for both numerator and denominator) for comparison across different diseases. The CER for stage IV esophageal cancer male patients was about 3.2 folds higher than that of stage I, or, US\$22,426 v.s. 6,987 (Table 4), indicating the significance of early diagnosis. Lifetime cost mentioned here is the reimbursement costs related to the patient's disease only, which did not include caregiver cost, transportation cost, nor costs from non-health care sectors (e.g. productivity loss, social services, and long term care, etc.), so the context of our analysis is essentially a narrower $^{32}$. Future studies are warranted to conduct a more comprehensive assessment from a societal perspective.

In conclusion, esophageal cancer in Taiwan appears to have slightly shifted into younger ages groups and incidence is still increasing. The lifelong health impacts (lifetime risks, LEs after diagnosis, EYLL) and financial burdens (lifetime cost, \& cost-effectiveness ratio) on healthcare sectors of esophageal cancer in Taiwan were quantified, which indicate the significance of developing effective strategies for prevention, early diagnosis and timely treatment for a more sustainable NHI. Future studies are warranted to explore the relative cost-effectiveness for various control strategies of this cancer.

\section{Materials and Methods}

The study was approved by the Institutional Review Board of National Cheng Kung University Hospital (IRB number: B-ER-104-103) before commencement, which followed the ethical principles of WMA (World Medical Association)and Declaration of Helsinki (https://www.wma.net/policies-post/ wma-declaration-of-helsinki-ethical-principles-for-medical-research-involving-human-subjects/). Data related to quality of life were collected after each patient provided informed consent.

Study population and datasets. All the patients' personal information was protected by encrypting their identification numbers, and all the analyses described below were performed in accordance with the relevant guidelines and regulations, and conducted in a secured area administered by the Health and Welfare Data Science Center, Ministry of Health and Welfare of Taiwan (https://dep.mohw.gov.tw/DOS/sp-GS-113.html?Query and https://dep.mohw.gov.tw/DOS/sp-GS-113.html?Query). Only summary tables could be brought out after verification by the officials to assure that there was no leakage of personal information.

Esophageal cancer included ICD-10-CM codes: C153, C154, C155, C158, C159, and ICD-O-3 morphology code: 8050/03 8089/3(squamous cell carcinoma), 8140/3(adenocarcinoma), and others. The stages of all esophageal cancer patients were stratified by pathological stage. If there was no pathologic staging information, the clinical stage was used. We interlinked the following 3 datasets, as summarized in Fig. 2: The Taiwan Cancer Registry from 2008 to 2014, the reimbursement database of the NHI system (2008-2015), and the Taiwan Mortality Registry (2008-2015) to ascertain every patient's current survival state. A total of 14,420 esophageal cancer patients with histopathological proof aged between 30 and 99 years old were retrieved for patients' demographics, date of diagnosis, cancer site, histology, and health care expenditure covered by the NHI. As cancer is a catastrophic illness, all costs of outpatient clinic visits and hospitalization, including laboratory and imaging studies and prescription medicines were covered by the NHI and waived from copayment. If patients were still alive at the end of December 2015, they were censored.

Estimation of lifetime risk by Cumulative incidence Rate (CIR). We calculated the age- and sex-specific incidence rates and CIR for the esophageal cancers. The numerators were the age-, sex-specific number of new cases from the Taiwan Cancer Registry for 2008-2010, 2011-2012, 2013-2014, and the corresponding populations at risk were abstracted from vital statistics as the denominators. The CIR was calculated from ages 30 to $84\left(\mathrm{CIR}_{30-84}\right)$ to estimate the lifetime risk of this specific cancer. The $\mathrm{CIR}_{30-84}$ was calculated from 2008 to 2014 to determine the trends in changes of lifetime risks. The formula is as follows ${ }^{33}$ :

$$
\mathrm{CIR}_{30-84}=1-\exp \left(-\sum\left(\mathrm{IR}_{\mathrm{i}}\right)\left(\Delta \mathrm{t}_{\mathrm{i}}\right)\right), \text { where } i=30-34,35-39, \ldots, 80-84^{\mathrm{i}}
$$

where $I_{i}$ is the incidence rate for the $i$-th age group and $\Delta t_{i}=5$ year age range.

Estimation of lifetime survival function, LE after diagnosis, and EYLL. The survival status of each esophageal cancer patient was acquired from the National Mortality Registry during 2008-2015. The survival functions were calculated with the Kaplan-Meier estimation method until the end of follow-up.

The extrapolation of the survival function was estimated using a rolling extrapolation algorithm, aided by the age- and sex-matched referents simulated from the National Vital Statistics life tables, as detailed in previous literature $^{19,34}$. The method could be briefly summarized as follows: First, for every patient in our cohort, we generated the age-, sex-, and calendar year of diagnosis-matched referents based on the life tables of Taiwan during 


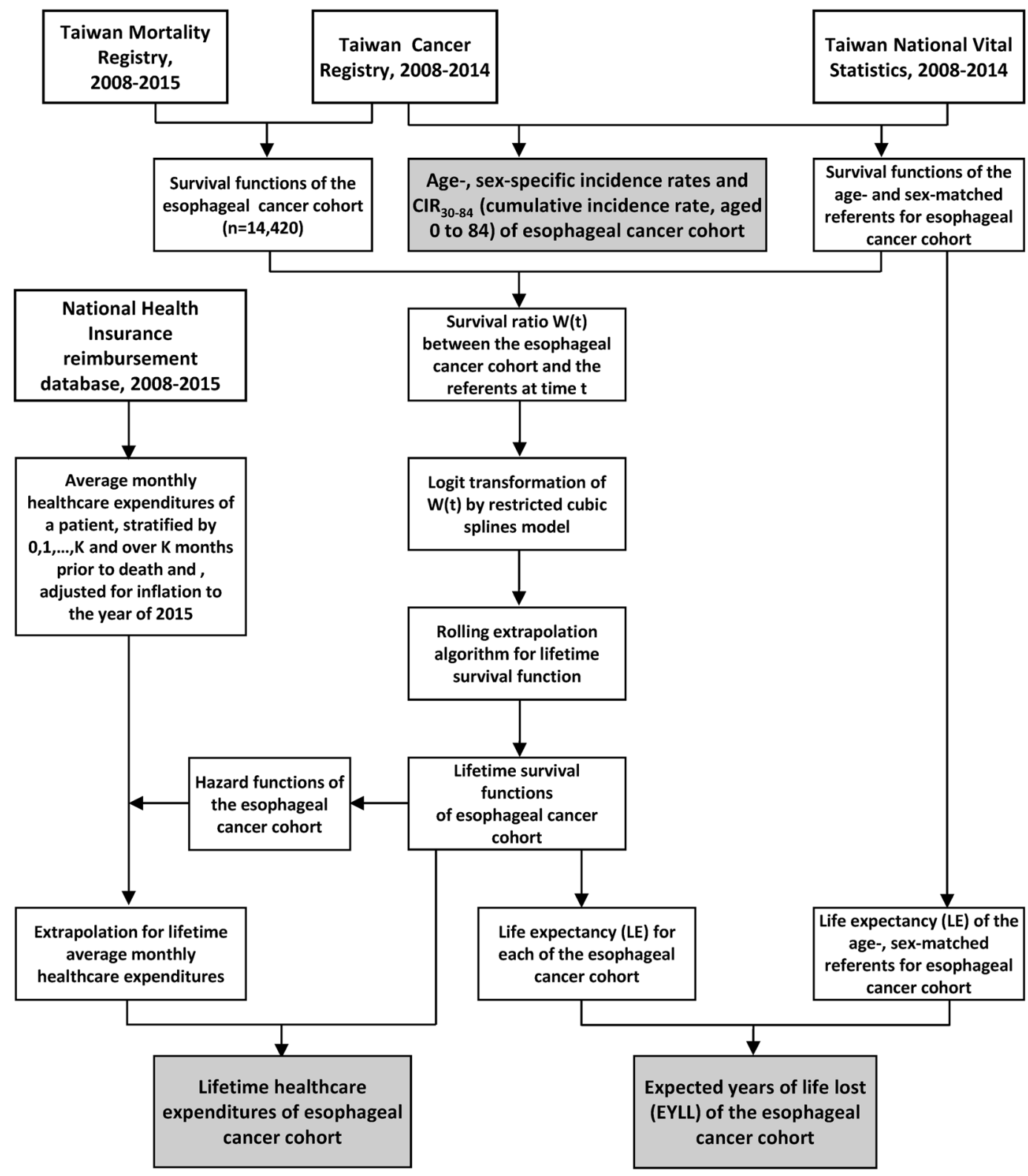

Figure 2. Flow diagram of the establishment of the Taiwan esophageal cancer cohort and retrieval of relevant data for the estimation of lifetime risks and outcomes.

the study period and estimated the Kaplan-Meier's lifetime survival function. Namely, we applied the hazard rates of the life table of the same calendar year for each individual patient to simulate the lifetime survival function of matched referents after the corresponding age, as all the blackish curves of Fig. 1 indicated. Second, we calculated the survival ratio between the cohorts of esophageal cancer and the referents at each time point and performed logit transformation of this ratio. Third, we fitted the logit-transformed relative survival curve into a restricted cubic splines model to extrapolate the survival function for one extended month by assuming that the fitted curve would be linear within the extrapolated one month, as shown in Fig. 3. By repeatedly perform the above procedure month-by-month (i.e., rolling over), we extrapolated the survival function of patients with esophageal cancer to their lifetimes, as shown in Fig. 1 after indicated end of follow-up. Because LE is the summation of the area under the lifetime survival curve, we were able to obtain the EYLL by comparing the LEs between the cancer cohort and that of the correspondingly matched referents, namely, the loss of life expectancy. The iSQoL 2 software was used to compute these estimations ${ }^{35}$.

Estimation of average monthly cost and its extrapolation to lifetime. As mentioned above, all costs of outpatient clinic visits and hospitalization of esophageal cancer patient were covered by the NHI. Therefore, our reimbursement data for this cancer was very comprehensive. 


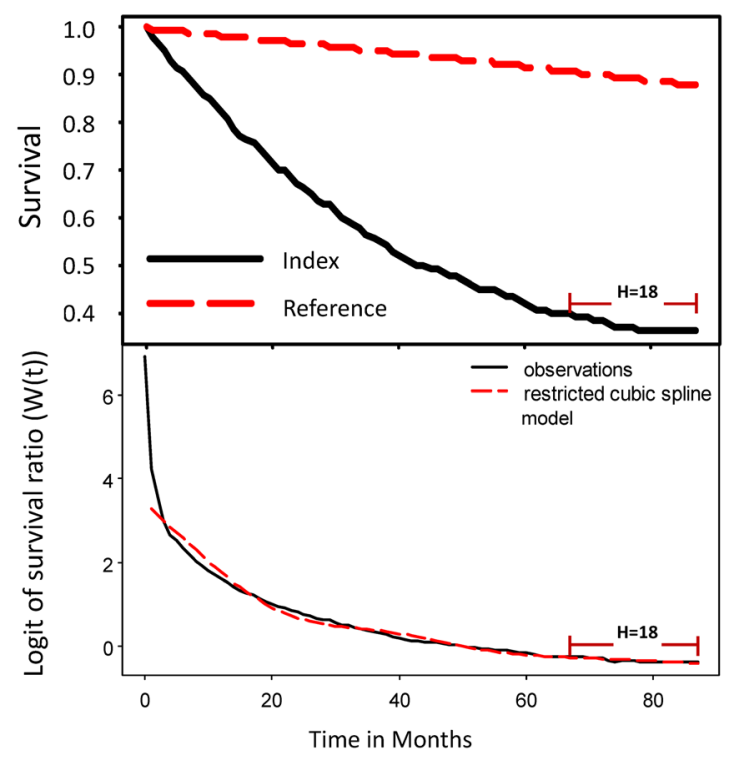

Figure 3. The upper panel shows survival functions of the index cohort (esophageal cancer, the blackish curve) and sex, age, and calendar-year matched reference cohort (the reddish dashed curve) simulated from vital statistics. The relative survival $W(t)$ is defined as the ratio of survival functions between the index group and reference population, namely, $S_{1}(t) / S_{0}(t)$. The lower panel shows the fitted restricted cubic spline model of the logit of $W(t)$ up to the end of follow-up, which fits well with the actual survival curve and the model of the last 18 months is linear (indicated as $\mathrm{H}=18$ ). With this part of the curve, we extrapolated one month by assuming that it would be still linear within the next one month. Then, we refitted the model again by adopting the extrapolated one month as the observed one and further rolling over another one more month, etc.

We summed up all the reimbursement costs for every esophageal cancer case and calculated the monthly average cost after diagnosis. The estimated lifetime cost was quantified by adding the product of monthly survival rates and monthly mean costs ${ }^{34}$. Briefly, since we assumed that the medical expenditures spent near mortality increased when the patient with esophageal cancer approached the end of life, we stratified the healthcare costs of those who died during the study period and those who were censored. We then analyzed the real data of those who died during the follow-up period to determine the specific month of when such costs began to increase approaching death and the increasing trend over time. Then, the monthly costs at time $t$ beyond the maximum follow-up time was calculated by a weighted sum of costs who were alive, costs who were near deaths, and costs who died on that month, where weights were the corresponding probabilities of survival at time $t$, death at a specific number of months after $t$, and death at time $t$, respectively ${ }^{34}$.

Received: 23 January 2019; Accepted: 4 February 2020;

Published online: 28 February 2020

\section{References}

1. Health Promotion Administration Ministry of Health and Welfare of Taiwan. Taiwan Cancer Registry Annual Report (2015). Taiwan: Department of Health, Executive Yuan, https://www.hpa.gov.tw/Home/Index.aspx, (Health Promotion Administration, Taipei, Taiwan, 2017).

2. Chiang, C. J. et al. Incidence and survival of adult cancer patients in Taiwan, 2002-2012. J. Formos. Med. Assoc. 115, 1076-1088 (2016).

3. Chiang, C. J. et al. Cancer trends in Taiwan. Jpn. J. Clin. Oncol. 40, 897-904 (2010).

4. Wang, Q. L., Xie, S. H., Wahlin, K. \& Lagergren, J. Global time trends in the incidence of esophageal squamous cell carcinoma. Clin. Epidemiol. 10, 717-728 (2018).

5. Alsop, B. R. \& Sharma, P. Esophageal Cancer. Gastroenterol. Clin. North Am. 45, 399-412 (2016).

6. Chu, P. C., Wang, J. D., Hwang, J. S. \& Chang, Y. Y. Estimation of life expectancy and the expected years of life lost in patients with major cancers: extrapolation of survival curves under high-censored rates. Value Health 11, 1102-1109 (2008).

7. Brown, M., Lipscomb, J. \& Snyder, C. The burden of illness of cancer: economic cost and quality of life. Annu Rev Public Health. 22, 91-113 (2001).

8. Zhang, Y. et al. Circular RNAs: emerging cancer biomarkers and targets. J. Exp. Clin. Cancer Res. 36, 152 (2017).

9. Xia, W. et al. Circular RNA has_circ_0067934 is upregulated in esophageal squamous cell carcinoma and promoted proliferation. Sci. Rep. 6, 35576 (2016).

10. Sanders, G. D. et al. Recommendations for Conduct,Methodological Practices, and Reporting of Cost-effectiveness Analyses Second Panel on Cost-Effectiveness in Health and Medicine. JAMA 316(10), 1093-1103 (2016).

11. Wen, C., Tsai, S. \& Chung, W. A 10-year experience with universal health insurance in Taiwan: measuring changes in health and health disparity. Ann Intern Med. 148, 258-267 (2008).

12. Lee, Y. C. et al. The impact of universal National Health Insurance on population health: the experience of Taiwan. BMC Health Serv. Res. 10, 225 (2010).

13. Chen, M. F., Yang, Y. H., Lai, C. H., Chen, P. C. \& Chen, W. C. Outcome of patients with esophageal cancer: a nationwide analysis. Ann. Surg. Oncol. 20, 3023-3030 (2013).

14. Cheng, Y. F. et al. Esophageal squamous cell carcinoma and prognosis in Taiwan. Cancer Med, 4193-4201 (2018). 
15. Chen, M. F., Chen, P. T., Lu, M. S., Lee, C. P. \& Chen, W. C. Survival benefit of surgery to patients with esophageal squamous cell carcinoma. Sci. Rep. 7, 46139 (2017).

16. Chen, S. C. et al. Secondary primary malignancy risk among patients with esophageal cancer in Taiwan: a nationwide populationbased study. Plos One 10, e0116384 (2015).

17. Lu, C. L. et al. Increasing trend of the incidence of esophageal squamous cell carcinoma, but not adenocarcinoma, in Taiwan. Cancer Causes Control 21, 269-274 (2010).

18. Rothman, K., Greenland, S. \& Lash, T. Modern Epidemiology. 3rd eds. Greenland, S. \& Lash, T. L., editors. Philadelphia: Wolters Kluwer Health/Lippincott Williams \& Wilkins, (Wolters Kluwer Health/Lippincott Williams \& Wilkins, 2012).

19. Wu, T. Y., Chung, C. H., Lin, C. N., Hwang, J. S. \& Wang, J. D. Lifetime risks, loss of life expectancy, and health care expenditures for 19 types of cancer in Taiwan. Clin. Epidemiol. 10, 581-591 (2018).

20. Kauppila, J. H., Mattsson, F., Brusselaers, N. \& Lagergren, J. Prognosis of oesophageal adenocarcinoma and squamous cell carcinoma following surgery and no surgery in a nationwide Swedish cohort study. Bmj Open, e021495 (2018).

21. Yang, B. et al. Prognostic significance of NFIA and NFIB in esophageal squamous carcinoma and esophagogastric junction adenocarcinoma. Cancer Medicine 7(5), 1756-1765 (2018).

22. Gardner, J. \& Sanborn, J. Years of potential life lost (YPLL)-what does it measure? Epidemiology. 1, 322-329 (1990).

23. Lapostolle, A., Lefranc, A. \& GremyI, S. A. Measure of premature mortality: comparison of deaths before age 65 and expected years of life lost. Rev Epidemiol Sante Publique. 56, 245-252 (2008).

24. Ferronha, I., Bastos, A. \& Lunet, N. Prediagnosis lifestyle exposures and survival of patients with gastric cancer: systematic review and meta-analysis. Eur. J. Cancer Prev. 21, 449-452 (2012).

25. Kuang, J. J. et al. Smoking Exposure and Survival of Patients with Esophagus Cancer: A Systematic Review and Meta-Analysis. Gastroenterol. Res. Pract. 2016, 1-9 (2016).

26. Chuang, S. C. et al. Risk of second primary cancer among patients with head and neck cancers: A pooled analysis of 13 cancer registries. Int. J. Cancer 123, 2390-2396 (2008).

27. Chen, S. H., Chan, S. C., Chao, Y. K. \& Yen, T. C. Detection of synchronous cancers by fluorodeoxyglucose positron emission tomography/ computed tomography during primary staging workup for esophageal squamous cell carcinoma in Taiwan. Plos One 8, e82812 (2013).

28. Poon, R. T. P., Law, S. Y. K., Chu, K.-M., Branicki, F. J. \& Wong, J. Multiple Primary Cancers in Esophageal Squamous Cell Carcinoma: Incidence and Implications. Ann. Thorac. Surg. 65, 1529-1534 (1998).

29. Zakko, L., Lutzke, L. \& Wang, K. K. Screening and Preventive Strategies in Esophagogastric Cancer. Surg. Oncol. Clin. N. Am. 26, 163-178 (2017).

30. Roshandel, G. et al. Endoscopic screening for esophageal squamous cell carcinoma. Arch. Iran. Med. 16, 351-357 (2013).

31. Marseille, E., Larson, B., Kazi, D. S., Kahn, J. G. \& Rosen, S. Thresholds for the cost-effectiveness of interventions: alternative approaches. Bull. World Health Organ. 93, 118-124 (2015).

32. Sanders, G. D. et al. Recommendations for Conduct, Methodological Practices, and Reporting of Cost-effectiveness Analyses: Second Panel on Cost-Effectiveness in Health and Medicine. JAMA 316, 1093-1103 (2016).

33. Day, N. A. A new measure of age standardized incidence, (Lyon: International Agency for Research on Cancer (IARC Scientific Publications No. 15) (1976).

34. Hwang, J. S., Hu, T. H., Lee, L. J. \& Wang, J. D. Estimating lifetime medical costs from censored claims data. Health Econ. 26, e332-e344 (2017)

35. iSQoL2. iSQoL2 Package for Windows [webpage on the Internet] (2017).

\section{Acknowledgements}

We are indebted to Dr. Wei-Lun Chang, and Forn-Chia Lin and Prof. Jing-Hsiang Hwang for their inputs and statistical consultation of this study. This work was supported partially by the National Cheng Kung University Hospital, Tainan, Taiwan [NCKUH-10709005]; and the Ministry of Science and Technology, Taiwan [MOST 1082627-M-006-001, MOST 107-2627-M-006-007].

\section{Author contributions}

Wu-Wei Lai wrote the main manuscript text. Chia-Ni Lin prepared Figures 1 and 2, and Tables 1, 2 and 4. ChaoChun Chang prepared the Table 3. Jung-Der Wang designed the whole experiment and manuscript re-checking. All authors reviewed the manuscript.

\section{Competing interests}

The authors declare no competing interests.

\section{Additional information}

Supplementary information is available for this paper at https://doi.org/10.1038/s41598-020-60701-3.

Correspondence and requests for materials should be addressed to J.-D.W.

Reprints and permissions information is available at www.nature.com/reprints.

Publisher's note Springer Nature remains neutral with regard to jurisdictional claims in published maps and institutional affiliations.

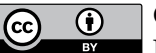

Open Access This article is licensed under a Creative Commons Attribution 4.0 International

License, which permits use, sharing, adaptation, distribution and reproduction in any medium or format, as long as you give appropriate credit to the original author(s) and the source, provide a link to the Creative Commons license, and indicate if changes were made. The images or other third party material in this article are included in the article's Creative Commons license, unless indicated otherwise in a credit line to the material. If material is not included in the article's Creative Commons license and your intended use is not permitted by statutory regulation or exceeds the permitted use, you will need to obtain permission directly from the copyright holder. To view a copy of this license, visit http://creativecommons.org/licenses/by/4.0/.

(C) The Author(s) 2020 